\title{
COMMENT ENVISAGE-T-ON D'ÊTRE SAGE-FEMME QUAND ON EST UN HOMME ?
}

\author{
L'intégration professionnelle des étudiants hommes sage-femmes
}

\section{Philippe Charrier}

La Découverte | «Travail, genre et sociétés »

2004/2 N $12 \mid$ pages 105 à 124

ISSN 1294-6303

ISBN 2747572102

Article disponible en ligne à l'adresse :

https://www.cairn.info/revue-travail-genre-et-societes-2004-2-page-105.htm

Distribution électronique Cairn.info pour La Découverte.

(C) La Découverte. Tous droits réservés pour tous pays.

La reproduction ou représentation de cet article, notamment par photocopie, n'est autorisée que dans les limites des conditions générales d'utilisation du site ou, le cas échéant, des conditions générales de la licence souscrite par votre établissement. Toute autre reproduction ou représentation, en tout ou partie, sous quelque forme et de quelque manière que ce soit, est interdite sauf accord préalable et écrit de l'éditeur, en dehors des cas prévus par la législation en vigueur en France. Il est précisé que son stockage dans une base de données est également interdit. 


\title{
COMMENT ENVISAGE-T-ON D'ÊTRE SAGE-FEMME QUAND ON EST UN HOMME ?
}

\section{L'INTEGRATION PROFESSIONNELLE DES ETUDIANTS HOMMES SAGE-FEMMES}

\author{
Philippe Charrier
}

I 1 existe une nouvelle dynamique dans la répartition des sexes au sein de la profession de sage-femme. L'ouverture récente de ce métier aux hommes pourrait entraîner une pluralité des modes d'exercice en fonction du sexe, autrement dit une segmentation professionnelle. La présence d'hommes dans ce milieu hyper féminisé - dont on verra qu'elle devrait augmenter dans les années à venir - se heurte à deux éléments fondamentaux liés précisément à l'univers féminin : l'activité porte sur l'espace le plus intime du corps de la femme et sur une expérience que seules les femmes peuvent connaître, l'enfantement.

Aujourd'hui, la grande majorité des professions et des métiers en France sont destinés à accueillir des personnes des deux sexes, certes en proportion variable selon les cas (Nicole Gadrey, 1992). Mais cela n'exclut pas une pluralité des modes d'exercice selon le sexe. Cela donne lieu parfois à des renversements dans la proportion de femmes dans un métier réputé masculin (Collin, 1994 ; Boigeol, 1993). Les rapports sociaux de sexe comportent souvent des enjeux sociaux sous-jacents. Par exemple, les débats autour de l'association "féminisation/dévalorisation" sont 
révélateurs de crises au sein des groupes professionnels concernés, que ce soit en termes de concurrence entre les groupes sociaux et de sexe ou de remise en question des hommes au plan symbolique et pratique (CacouaultBitaud, 2001).

Or, la profession de sage-femme s'ordonne principalement autour des valeurs liées à la féminité. L'apparition de praticiens masculins aura vraisemblablement des conséquences sur l'identité professionnelle. C'est donc plus largement cette dernière question qui se trouve posée (Dubar, 1996).

Pour rendre compte de cette évolution, nous emprunterons les concepts de "career" et de "segments professionnels" développés par la sociologie interactionniste des groupes professionnels (Bucher et Strauss, 1992 ; Hughes, 1996). L'étude sur laquelle nous nous appuyons traitant des futurs hommes sages-femmes, ces deux concepts sont adaptés pour rendre compte du parcours biographique qui les a amenés à embrasser cette profession et des représentations qu'ils s'en font. Il faudra, en outre, évaluer s'ils se situent ou non dans une logique d'homogénéisation professionnelle, à savoir s'ils envisagent leur exercice professionnel de manière identique aux femmes sages-femmes, du point de vue du mode d'exercice, de l'implication dans la profession, du choix de carrière, etc. Pour cela, nous avons analysé le discours d'étudiants hommes sages-femmes et la manière dont ils envisagent leur avenir professionnel.

Mais avant de développer cette analyse, il convient de rappeler le parcours historique de cette profession en France, puis de revenir sur les éléments nouveaux qui pourraient remettre en cause le schéma interne de la répartition entre hommes et femmes.

\section{ÉVOLUTIONS HISTORIQUES ET PERSPECTIVES : LA QUESTION DU GENRE CHEZ LES SAGES- FEMMES}

\section{Quelques jalons historiques sur la profession de sage-femme}

Cette profession - dont il est couramment admis qu'elle est l'une des plus anciennes - a été l'objet d'une lutte entre deux groupes, les chirurgiens d'une part, les matrones d'autre part. Soulignons que cette opposition recouvre une distinction entre deux populations, l'une féminine et l'autre masculine. Les matrones ont eu jusqu'à l'avènement des "Lumières" le monopole de l'accompa- 
gnement des naissances, monopole qu'elles ont dû céder aux chirurgiens et à la médecine clinique naissante. Ces matrones tiraient leur légitimité professionnelle non seulement de leurs connaissances de l'accouchement mais aussi d'une institution, l'Eglise, qui leur accorde une forme de sacralité (Laget, 1982). Elles sont chargées de la pratique de l'ondoiement, un rite destiné à tenir lieu de baptême lorsqu'un danger de mort (de l'enfant) est imminent. Cette charge, à forte portée symbolique, s'accompagnait de l'idée que seule une femme pouvait accomplir cet acte. Dès lors, était entérinée l'idée que l'accouchement était une "affaire de femmes", d'où le rôle primordial du sentiment d'empathie.

Progressivement, cette évolution vers une reconnaissance de la fonction de "matrone" a provoqué un changement sémantique: on parle désormais de sage-femme. Parfois soupçonnée de pratique païenne, la "matrone" est contrôlée par l'autorité de l'Eglise puisqu'elle est assermentée par l'évêque.

L'État va ensuite intervenir dans le contrôle des naissances et par conséquent sur les sages-femmes. Au XVIIIe siècle, faisant le constat que trop de femmes et d'enfants meurent lors des accouchements, notamment en raison d'une méconnaissance supposée des sages-femmes, le pouvoir royal intervient en impulsant une politique nataliste. A partir du milieu du XVIIIe siècle, les chirurgiens définissent un "art de l'accouchement" bien éloigné des connaissances pragmatiques et traditionnelles des sages-femmes. Comme l'a décrit Jacques Gelis, chacune de ces corporations luttait l'une contre l'autre, selon deux rationalités bien différentes (Gelis, 1988). Dans ce "combat" de légitimité, l'issue a donné raison aux chirurgiens. Ce sont les premiers chirurgiens-obstétriciens, des hommes par conséquent, qui vont prendre en charge la formation des sages-femmes. A partir de là, se développe la "subordination" professionnelle des sages-femmes aux médecins. Comme le souligne Danièle Carricaburu, "en deux siècles, les chirurgiens accoucheurs ont réussi à déposséder les femmes de leur monopole de la naissance" (Carricaburu, 1994).

Pourtant au XIXe siècle, la sage-femme est une personnalité incontournable dans les villages ou les bourgs. En plus de ses tâches relatives à l'accouchement, elle intervient dans la prime éducation de l'enfant, rendant des services proches de ceux de l'infirmière ou de l'assistante sociale. Au siècle suivant, la spécialisation de ces fonctions et la concurrence des infirmières, des assistantes sociales et des médecins, entraînent une chute du nombre de sages-femmes mais aussi une baisse de leur prestige 
${ }^{1}$ En fait, cet Ordre des Sages-Femmes est pensé sous le Régime de Vichy et sera pour ainsi dire entériné à la fin de la guerre, en 1945.

${ }^{2}$ Nous suivons ici la définition de

Claude Dubar et Pierre Tripier, qui voit dans le terme de profession, à la fois une identité collective, une position, une spécialisation et une classification. C'est sur le plan de la profession comme identité collective que porte notre étude. Claude Dubar, Pierre Tripier, Sociologie des professions, Paris, A. Colin, 1998, pp.8-13.

\footnotetext{
${ }^{3}$ Pour un point de vue général sur ces questions, voir par exemple, Nicole Gadrey, op. cit., 1992.

${ }^{4}$ La profession est "ouverte" aux hommes depuis 1982. Les premiers hommes sagefemme diplômés l'ont été en 1985.

${ }^{5}$ Accouchement normal, par opposition à dystocie.

${ }^{6}$ Accouchement rendu difficile en raison d'une anomalie maternelle ou fœtale. Par exemple, l'accouchement par césarienne est défini comme dystocique.
}

social. Il faut attendre la fin de la seconde guerre mondiale et la mise en place effective de l'ordre des sagesfemmes ${ }^{1}$ pour qu'il y ait de leur part une volonté de reconquête.

Enfin, le dernier volet historique de cette profession tient dans une forte évolution du mode d'exercice. Pratiquant en majorité en libéral, les sages-femmes se sont dirigées en masse vers l'hôpital, dès les années 1950, lieu où les accouchements se sont progressivement multipliés en France. Il en découle une hausse continue du salariat dans cette profession, qui culmine aujourd'hui aux alentours de $85 \%$. Cela leur a été sans doute bénéfique pour marquer une frontière entre la vie privée et la vie professionnelle, pour assurer un revenu stable et accroître certaines compétences techniques. Mais la contrepartie est une perte d'indépendance par rapport aux médecins et à l'administration. Or, cette volonté d'indépendance, est aujourd'hui au cœur des revendications des sages-femmes, notamment celles exprimées lors du conflit d'avril et mai 2001.

\section{Les perspectives autour de la question du genre chez les sages-femmes}

On observe plusieurs dynamiques dans la répartition sexuée dans la plupart des professions². Même les professions les plus "masculines", comme la magistrature ou les ingénieurs voient leurs effectifs se féminiser, parfois de manière spectaculaire (Marry, 1989 ; Boigeol, 1993). La plupart de ces recherches mettent en avant les difficultés, les obstacles ou les inégalités auxquels ces femmes sont confrontées dans leur insertion professionnelle puis dans leur évolution de carrière ${ }^{3}$, les tendances à la segmentation professionnelle (Boigeol, 1993), et le recours aisé à une logique de dévalorisation ou de revalorisation professionnelle (Cacouault-Bitaud, 2001). En revanche, les travaux sur la masculinisation d'une profession sont rares (Muel-Dreyfus, 1983 ; Alonzo, 1996).

A cet égard, la profession de sage-femme est intéressante dans la mesure où elle accueille désormais des hommes ${ }^{4}$, bien qu'elle reste marquée par la forte présence des femmes et bien qu'une logique sexuée joue entre sages-femmes et gynécologues-obstétriciens puisque ces derniers restent en majorité des hommes.

En structure hospitalière, le travail autour de l'accouchement est divisé en deux champs d'activité : l'eutocie ${ }^{5}$ pour les sages-femmes et la dystocie ${ }^{6}$ pour les gynécologues-obstétriciens. L'hospitalisation croissante des naissances a créé une division du travail suivant une distinc- 
tion entre normalité et anormalité physiologique et médicale. Cette différenciation en recoupe une autre, celle du genre. Même de nos jours, bien que les sages-femmes assurent la très grande majorité des accouchements (75\%), bien qu'elles jouissent d'un statut de profession médicale, qu'elles bénéficient d'un statut médical, défini par le Code de la Santé Publique, "elles sont pourtant mal connues et socialement peu considérées" (Carricaburu, 1994). Enfin, la dernière caractéristique des sages-femmes réside dans une part non négligeable d'exercice à temps partiel $(30 \%)$, alors qu'il y a dix ans, cette proportion était moitié moindre (DRESS, 2001).

En France, il existe seulement une centaine d'hommes sages-femmes pour plus de quinze-mille praticiennes. Mais la venue $\mathrm{d}^{\prime}$ hommes dans la profession va sans doute s'accroître en raison d'un mouvement initié en partie par l'intermédiaire de la formation. Une forme de recrutement originale a cours à l'école de Grenoble, et semble devoir être étendue à l'horizon 2003 à toutes les écoles de sages-femmes ${ }^{7}$.

\section{Un recrutement original à l'Ecole de sages-femmes de Grenoble}

Nous avons remarqué, lorsque nous avons dispensé des cours dans cette école, la présence de plusieurs étudiants hommes, ce qui peut d'abord surprendre.

Cette originalité s'explique en partie par un mode de recrutement spécifique à Grenoble, les étudiants étant recrutés après une première année de médecine, alors qu'ailleurs il s'agit d'un concours ouvert à toute personne ayant le baccalauréat ou un niveau équivalent. Dans ce cas, l'étudiant classé entre la quatre-vingtième et la centième place à l'examen PCEM1 (reçu mais "collé"8) se voit proposer la carrière de sage-femme". En conséquence, des filles mais aussi des garçons ayant réussi leur première année de médecine, mais exclus par le numerus clausus tant en médecine qu'en odontologie, peuvent entreprendre des études de sage-femme. Le cas isérois est un véritable laboratoire, puisque le système est en place depuis 1992. Dès lors, des hommes ont intégré cette formation à Grenoble depuis dix ans.

$\mathrm{Du}$ point de vue des formateurs et des sages-femmes enseignantes, il s'agit d'un atout. En effet, ces étudiants, ayant parfois effectué deux, voire trois premières années de médecine, leur apparaissent bien mieux préparés aux enseignements médicaux, que des étudiants sortant d'un baccalauréat généraliste ${ }^{10}$. Cette recherche d'un "meilleur niveau" peut aussi s'expliquer par la lutte entre les
7 Il s'agit là d'une des conséquences du dernier conflit des sages-femmes. Partant de l'idée que la profession jouit du statut médical, il conviendrait donc de l'intégrer à la formation médicale. A la suite d'un arrêté du 24 juillet 2001, les étudiants doivent avoir validé l'examen classant de fin de 1ère année de PCEM1.

8 Etudiant ayant réussi le concours mais avec une moyenne insuffisante pour entrer dans le quota de places alloué à la faculté de médecine.

\footnotetext{
${ }^{9}$ Bien entendu, si l'étudiant est reçu en médecine ou en odontologie, rien ne l'empêche de choisir sagefemme. Toutefois, parmi les étudiants sur lesquels s'appuie cette étude, aucun n'était dans ce cas. Ils étaient tous confrontés au choix suivant : école de sages-femmes ou réorientation.

10 Ceci est confirmé dans un reportage du Monde de l'édition du 6 novembre 2001 portant sur l'école de sages-femmes de Grenoble : "Donnant une large place aux matières médicales plutôt que scientifiques, le contenu de la première année des études médicales à Grenoble s'adapte assez bien au recrutement des sages-femmes. "On arrive à l'école avec une vraie culture médicale sur l'anatomie, la physiologie, on est moins perdu", considère Chantal, étudiante en deuxième année.
} 
${ }^{12}$ Ce chiffre est celui des hommes inscrits à l'Ordre des sagesfemmes en 2001. Cette inscription se fait automatiquement lors de l'obtention du diplôme. Cela signifie que certains membres peuvent être inscrits sans pour autant exercer, ou bien exercer une autre profession, grâce à des équivalences (nous pensons aux infirmiers). Par ailleurs, nous avons effectué un recensement des hommes sages-femmes en exercice qui fait état d'une centaine de diplômés depuis 1985 dont six n'exercent plus cette profession. Ces écarts viennent sans doute des élèves nouvellement diplômés et non encore répertoriés par l'Ordre, mais aussi du fait que certains sages-femmes n'ont pas signalé leur changement de lieu de travail.

\footnotetext{
${ }^{13}$ Nous n'avons pas tenu compte de l'effectif à l'école de Grenoble, ce qui aurait entraîné un biais. A titre indicatif, il y avait à la même époque 12 garçons pour un effectif total de 85 étudiants.

Cinq des écoles contactées n'ont pas souhaité nous transmettre les chiffres de leurs effectifs.
}

médecins et les sages-femmes dans le champ professionnel de la naissance. Initiés aux études médicales, les étudiants sages-femmes ont souvent une moyenne bien supérieure à dix à l'examen PCEM1 et leur légitimité s'en trouve renforcée.

\section{Quelques conséquences envisageables}

La principale conséquence que l'on peut envisager, dès à présent, est l'augmentation substantielle d'hommes qui vont se voir proposer la carrière de sage-femme. D'après l'Ordre des sages-femmes, il existe soixante-quinze hommes pouvant exercer la profession de sage-femme ${ }^{11}$, alors qu'actuellement on dénombre en France 15027 sages-femmes en activité12. Tous les étudiants qui se verront proposer cette filière ne choisiront pas cette voie mais on peut penser qu'en moyenne deux ou trois étudiants par an pour chacune des écoles intègreront le cursus de sage-femme, comme c'est le cas à Grenoble. Autrement dit, à l'horizon 2006-2010, la proportion d'hommes dans cette profession devrait augmenter considérablement : on pourrait estimer entre soixante et quatre-vingt dix hommes diplômés par an pour environ un total de quatre cent soixante sages-femmes (si le nombre de diplômés reste constant), soit par hypothèse une proportion de 13 à 19\% d'hommes sur la totalité des diplômés.

Nous avons effectué un recensement des effectifs de vingt-quatre des trente écoles de sages-femmes en France métropolitaine en 2000 qui permet de faire l'état de la présence d'hommes en formation ${ }^{13}$.

\begin{tabular}{lcccc}
\hline $\begin{array}{l}\text { Années } \\
\text { d'études }\end{array}$ & $\begin{array}{c}\text { Nombre } \\
\text { total } \\
\text { d'étudiants }\end{array}$ & $\begin{array}{c}\text { Nombre } \\
\text { de garçons } \\
\text { inscrits }\end{array}$ & $\begin{array}{c}\text { Nombre } \\
\text { de filles } \\
\text { inscrites }\end{array}$ & $\begin{array}{c}\text { Pourcentage } \\
\text { de garçons (\%) }\end{array}$ \\
\hline $\begin{array}{l}\text { Première } \\
\text { année }\end{array}$ & 563 & 9 & 554 & 1,6 \\
\hline $\begin{array}{l}\text { Deuxième } \\
\text { année }\end{array}$ & 500 & 14 & 486 & 2,8 \\
\hline $\begin{array}{l}\text { Troisième } \\
\text { année }\end{array}$ & 484 & 7 & 477 & 1,4 \\
\hline $\begin{array}{l}\text { Quatrième } \\
\text { année }\end{array}$ & 452 & 11 & 441 & 2,4 \\
\hline Total & 1999 & 41 & 1958 & 2 \\
\hline
\end{tabular}

Si nous nous en tenons aux effectifs de quatrième année, avec cinq étudiants, l'école de Grenoble fait encore figure d'exception car cette population masculine représente $20 \%$ de l'effectif total (soit cinq sur une promotion

110 A Travail, Genre et Sociétés n 12 - Novembre 2004 
de vingt élèves). Ce taux est à rapprocher du taux général d'étudiants garçons inscrits dans le tableau ci-dessus $(2 \%)$. Cette comparaison montre combien le changement de recrutement peut faire évoluer la répartition professionnelle entre hommes et femmes.

\section{LE CONTEXTE DE L'ENQUÊTE}

L'étude portait sur les étudiants garçons inscrits à l'école de Grenoble lors de l'année universitaire 19992000. Ils étaient au nombre de douze : deux en première année, deux en deuxième année, trois en troisième année et cinq en quatrième année. Nous avons procédé par entretiens semi-directifs. Les thèmes abordés concernaient à la fois le rapport des étudiants aux sages-femmes dans le cadre de leurs stages, les relations avec les parturientes, leur vision du groupe professionnel, leurs représentations de l'avenir professionnel, et leur positionnement en tant que représentant d'un genre minoritaire. Cette enquête a été réalisée au cours des mois de juin et juillet 2000, en collaboration avec l'école de sages-femmes de Grenoble ${ }^{14}$.

Dans le but d'évaluer si une dynamique de segmentation était en cours, nous avons interrogé les étudiants sur la manière dont ils vivent et se représentent leur intégration professionnelle, il s'agissait d'appréhender les éléments contribuant à leur "carrière", en prenant particulièrement en compte leur position minoritaire. En outre, l'objectif était de faire émerger les logiques qui accompagnent cette vision de leur "carrière".

A ce titre, le débat qui a eu cours dans la profession concernant la dénomination de ces hommes sages-femmes, est révélateur puisque l'Ordre des sages-femmes a émis l'hypothèse de nommer différemment les hommes sages-femmes. Etymologiquement, le terme de sagefemme n'exclut pas le fait qu'il soit utilisé par des hommes, puisque la "femme" en question peut être celle sur laquelle le praticien intervient (la parturiente) comme nous l'ont fait remarquer certains hommes sagesfemmes ${ }^{15}$. L'Ordre des sages-femmes, avec l'aide de l'Académie Française, leur a proposé des dénominations parfois très étonnantes: "maïeuticiens" ou "parturologues". Toutefois, lors de notre enquête aucun des étudiants n'a exprimé la volonté d'endosser l'une de ces dénominations, bien au contraire. Certains acceptent d'être nommés "la sage-femme". Autrement dit, ce débat reflète plutôt un embarras de certaines sages-femmes qui ne
${ }^{14}$ Nous remercions particulièrement sa directrice, Danielle Ferrant, pour son aide.
${ }^{15}$ Odette Yvonne Pouvreau-Romilly, auteure sage-femme, soutient a contrario l'idée que sage-femme signifie "celle qui sait", autrement dit concerne la professionnelle. Cette divergence met en valeur celle qui exerce, la sage-femme, et donne alors un caractère sexué à la profession. Il ne s'agit pas ici de trancher sur une quelconque "vérité" étymologique, mais plutôt de souligner que ceci s'apparente à une sorte de rhétorique professionnelle. Odette Yvonne PouvreauRomilly, L'événement de la naissance, Toulouse, Eres, 1991. 
savent comment les incorporer dans la profession et comment ces derniers s'intègrent.

\section{DES HOMMES POSITIONNÉS ENTRE UN CHOIX PAR DÉFAUT ET DES COMPÉTENCES "FÉMININES"}

${ }^{16}$ Aux dires de ceux-ci, c'est également le cas pour la quasi-totalité des filles.
Les étudiants garçons sont confrontés à deux éléments incontournables. D'une part, leur arrivée dans le métier n'est pas le résultat d'une vocation et, d'autre part, ils doivent faire face à l'idée communément admise que cette profession serait une "profession de femmes"16.

\section{Un choix par défaut ou l'œuvre du hasard}

Pour tous ces garçons, entrer dans la profession de sage-femme n'était pas l'objectif défini lors de leur inscription en première année de médecine. On peut donc exclure toute logique de vocation pour les hommes sagesfemmes formés à Grenoble. La plupart des garçons se destinaient vaguement aux professions médicales, pour quelques-uns avec un objectif précis de spécialisation. Deux d'entre eux souhaitaient devenir obstétricien. Ces éléments biographiques induisent nécessairement l'accomplissement d'un deuil de la carrière médicale.

"Pendant le deuil de médecine, donc j'appelle ça le deuil de médecine, en première année bon, on est confronté aux services, donc dans les services infirmiers à des responsabilités des soins techniques et puis, tout l'univers de la psychologie qui est, qu'il doit y avoir dans le service hospitalier", Cédric, deuxième année.

Cet étudiant marque bien qu'au départ c'est plus par contrainte qu'il se dirige vers le métier de sage-femme. Il lui faut abandonner ses espoirs professionnels, qu'il se "fasse une raison" et qu'il se construise un autre avenir. Ainsi, on s'aperçoit de l'importance que peut avoir le vécu de la formation dans la construction de l'identité professionnelle, qui plus est lorsqu'elle n'est pas choisie. De même, cela confirme l'idée que la construction de l'identité professionnelle est un processus qui débute dès la formation. Par conséquent, il apparaît que la première année d'étude - essentiellement constituée de stages dits "infirmiers" et de cours généraux sur les soins et la médecine - est l'obstacle qu'il faut franchir pour accomplir ce travail de deuil. Lors de la deuxième année, offrant les premiers cours d'obstétrique et les stages en maternités, les garçons s'intègrent mieux dans leur formation et commencent à se faire une idée précise de leur futur métier. 
Avant l'intégration de l'Ecole, la connaissance de la profession est quasi nulle. Lorsqu'ils étaient encore étudiants en médecine, certains ont accepté l'éventualité d'être sage-femme sans connaître l'activité de cette profession, son étendue, ni même son statut de profession médicale. Pour tous, c'est d'ailleurs après deux ou trois tentatives en médecine qu'ils se sont orientés vers cette carrière ${ }^{17}$.

"Pourquoi j'ai choisi d'être sage-femme... ? A mon avis c'est plus... C'est plus dû aux résultats du concours de première année de médecine, plutôt que véritablement une vocation, quoi. J'crois que j'y avais pas trop pensé il $y$ a deux ans, un peu plus pensé quand j'ai appris qu'on pouvait intégrer l'Ecole de sages-femmes par le concours de médecine. Et après, ben, c'était même plus y penser, j'étais obligé vu mes résultats au concours de médecine, j'avais que cette solution. Donc pendant l'été ben, je me suis dit que le pas était pas très grand entre médecine et sage-femme. Donc je me suis dit que c'était peut-être la meilleure solution pour moi et la plus facile dans un premier temps. Et voilà. Je suis parti là-dedans un peu en me disant que bon, on verra, on verra comment ça se passe. Je suis pas parti en me disant c'est sûr que ça va me plaire. Bon c'est vrai, je savais pas comment ça allait se passer. Je me suis dit qu'on verrait bien, dans peu de temps, comment commencerait l'année quoi", Christophe, première année.

Il s'agit clairement d'un choix par défaut pour la totalité de ces élèves, tout au moins au départ. Ce choix s'apparente parfois à une contrainte comme l'explique Christophe. Selon eux, ils ont très peu, voire pas du tout, songé au fait qu'il s'agissait d'une profession extrêmement féminisée et que cela pouvait augurer de difficultés dans leur intégration professionnelle. Ainsi, la méconnaissance de la profession peut être un atout pour attirer des étudiants et effacer tous les obstacles potentiels, notamment celui du genre.

\section{Des hommes dans une profession de femmes}

Il reste que ces étudiants hommes vont intégrer une "profession féminine". Comme l'a souligné FrançoisXavier Schweyer, la profession de sage-femme se fonde en grande partie sur un ensemble de compétences "dites féminines": "Les compétences médicales basées sur un savoir technique ne sont pas les seules à être mentionnées pour fonder le système d'expertise de la profession. La dimension relationnelle, pédagogique, de conseil et même d'empathie, cette faculté à s'identifier à quelqu'un, à ressentir ce qu'il ressent, est citée comme qualité nécessaire à l'exercice du métier" (Schweyer, 1996). Si dans la pratique rien n'empêche les hommes d'exercer cette profession, ces compétences "dites féminines", qui fonctionnent selon une logique imaginaire voire idéologique, justifient l'exigence

\author{
17 Ce choix a été \\ souvent effectué au \\ dernier moment, c'est- \\ à-dire le jour même des \\ épreuves. C'est dans \\ ces instants que \\ l'étudiant doit exprimer \\ ses préférences, entre \\ médecine, odontologie \\ et sage-femme.
}


${ }^{18}$ Cet exemple montre bien le caractère imaginaire voire idéologique des compétences "dites féminines", puisque beaucoup de sagesfemmes débutent leur carrière sans avoir accouché elle-même.

${ }^{19}$ Dans ce dernier cas, quelques étudiants soulignent que les difficultés viennent plus de l'entourage de la parturiente (notamment de l'époux) que de la femme elle-même. de qualités qui sont propres à la femme et non à la professionnelle. Par exemple, l'empathie, cette faculté intuitive à percevoir ce que ressent et vit la parturiente lors de la grossesse et de l'accouchement (puisque la sage-femme l'a vécu ou peut le vivre) discrédite de facto les hommes ${ }^{18}$. Les dimensions relationnelle, pédagogique, de conseil, d'empathie, sont des caractères culturellement associés à la femme (Bourdieu, 1990) et réinvestis dans la rhétorique professionnelle (Paradeise, 1985) pour justifier la présence quasi-exclusive des femmes.

Les compétences "féminines", ces garçons ne peuvent pas la posséder ni même l'obtenir. Ils partent donc avec un "handicap", qui est un signe a priori d'incompétence. $C^{\prime}$ 'est dans le cadre de leurs stages de formation qu'ils rencontrent parfois des réticences de la part de praticiennes à travailler avec des hommes. Certaines sagesfemmes en exercice ne peuvent s'expliquer la présence de ces garçons. Selon elles, ils n'auraient pas les qualités requises (sous-entendu féminines) pour exercer ce métier. Cette opinion n'est pas partagée par toutes les sagesfemmes loin de là, et de l'aveu même des étudiants, des éléments comme l'âge de la personne ou bien l'ambiance au sein du service sont des facteurs explicatifs de cette "résistance aux hommes".

Enfin, les cas de refus de la part de la parturiente sont rares, sauf lorsque les convictions religieuses s'insèrent dans la relation ${ }^{19}$.

Ces constats plaident en faveur d'une présence très minoritaire des hommes dans cette profession (0,5\%). D'ailleurs, lors de nos premiers contacts auprès de sagesfemmes de la région grenobloise, il semble que la forme de recrutement qui a cours à Grenoble suscite quelques réprobations, notamment en raison de ses conséquences sur l'afflux d'hommes. Pour cette raison, on ne peut pas réellement parler de masculinisation. Toutefois, au vu du récent conflit des sages-femmes (avril-mai 2001), la question se pose tout de même à moyen terme.

\section{UNE LOGIQUE D'INTÉGRATION PROFESSIONNELLE ENTRAÎNANT UNE SEGMENTATION : ACCOUCHER SYMBOLIQUEMENT LA PROFESSION}

Passons aux discours des étudiants et voyons quelle est leur logique d'intégration professionnelle. Disons dès à présent que si elle est efficace, elle entraîne toutefois une distinction qui suit le découpage homme/femme et 
par conséquent introduit une segmentation en fonction du genre.

Ces étudiants sont, dès le début, confrontés dans leurs stages à des difficultés qui peuvent rendre plus problématique leur insertion professionnelle future et la représentation qu'ils en ont. Ce n'est pas tant dans la relation aux patientes, à l'intimité féminine qu'ils disent se heurter. S'ils identifient des tensions, elles proviennent de leurs relations avec une minorité des sages-femmes.

"C'est plutôt les sages-femmes sur les terrains de stages. C'était difficile à accepter. Il y en a certaines, un homme dans la profession non. Bon, elles travaillent en $\mathrm{CHU}$ donc elles ont un devoir de formation. Et nous on n'est pas hors-la-loi hein. L'homme a le droit de faire, d'être sage-femme depuis 1982, donc elles sont obligées de nous encadrer. Donc elles font l'encadrement. Mais faut voir l'ambiance quoi. Il y en a certaines avec qui, quand on allait en garde, c'était pas de gaieté d'cœur. Mais bon ça passe. Ça a jamais été très méchant. Enfin pour mon cas, j'ai jamais été saqué... Fallait être poli, fallait être gentil avec tout le monde et puis c'est tout quoi. Fallait laisser passer l'orage. Sinon fallait se dire que, qu'il y avait pas plus de tolérance chez une sage-femme. C'est quand même un peu ce qui m'a choqué quoi", Pierre, quatrième année.

Quelles stratégies ces jeunes hommes développent-ils face à ces tensions ?

Tout au long de leur formation, se dessine une logique de contournement symbolique des "compétences dites féminines". Elle peut se résumer de la manière suivante : à défaut de pouvoir posséder ces compétences, à défaut de pouvoir connaître réellement, par empathie, l'accouchement (et vivre l'expérience "irremplaçable" de la femme enceinte), ces hommes sages-femmes assurent symboliquement l'accouchement de la profession. Au-delà des compétences techniques et relationnelles que ces hommes acquièrent (n'oublions pas qu'ils sont formés exclusivement par des femmes), la plupart endossent un rôle maïeutique non seulement envers la parturiente mais aussi envers le groupe professionnel. Or, cette logique positionne de facto ces hommes dans un rôle qui les distingue de la grande majorité des sages-femmes.

Le mouvement de fond qui traverse le groupe professionnel est la volonté d'être reconnu par les acteurs administratifs, politiques, médicaux mais également par la population comme une profession à part entière. Le fait que l'Ordre des sages-femmes soit dirigé par une sagefemme depuis 1995 alors que jusque-là un médecin était à sa tête est un exemple de cette détermination. L'acquisition de la quatrième année d'études en est un autre. Le conflit d'avril-mai 2001 participe au mouvement, car il s'agit pour les sages-femmes de se positionner dans le champ de la naissance par rapport aux médecins géné- 
ralistes et aux gynécologues-obstétriciens. En définitive, l'autonomie qu'elles recherchent depuis longtemps comme le rappelle Danièle Carricaburu, elles espèrent la rencontrer en assumant pleinement leur statut de profession médicale (Carricaburu, 1987). Les sagesfemmes ne forment pas une profession "établie" au sens où l'entendait Everett $C$. Hughes mais tentent de réunir les conditions pour atteindre cet objectif (Hughes, 1996).

Dans le discours des étudiants, plusieurs thématiques viennent soutenir cette dynamique de professionnalisation dans laquelle ils souhaitent prendre place. La première passe par une exacerbation de la logique libérale de la profession. La deuxième tient au rôle que devrait assumer le genre masculin dans un tel système professionnel. La troisième concerne le rôle que certains responsables de la profession souhaitent leur faire endosser. Enfin, la dernière thématique porte sur l'intérêt que les étudiants accordent aux questions de fond qui secouent aujourd'hui la profession.

\section{La valorisation de 1'exercice libéral}

${ }^{20}$ Rappelons que ce discours a été recueilli avant le déclenchement du mouvement de grève.

${ }^{21}$ Pour ces étudiants, il y a une volonté manifeste d'étendre ce "noyau central" à la sphère de la naissance. Il s'agit là aussi d'un élément autorisant le contournement des compétences "dites féminines" très liées à l'accouchement, comme événement.

Le récent conflit de mars-avril 2001 a mis l'accent sur la volonté de reconnaissance professionnelle des sagesfemmes. Ce mouvement, que l'on peut expliquer par une volonté d'autonomisation du groupe professionnel par rapport aux autres professions de santé, trouve un écho dans le discours des étudiants hommes ${ }^{20}$.

A l'exception d'un cas, tous les étudiants expriment le souhait d'exercer en libéral, dans un délai d'environ cinq ans, après une période d'acquisition d'expériences qu'ils jugent nécessaires pour cette pratique. Il est remarquable que leur désir se porte sur la forme d'exercice la moins courante: en effet, l'activité libérale ne représente que $12,6 \%$ des sages-femmes. De plus, nombreuses sont celles qui exercent en suites de couches ou en rééducation postpartum, ce qui ne correspond pas au cœur de l'activité, c'est-à-dire l'accouchement (DRESS, 2001) ${ }^{21}$. Il faut dire que non seulement ces hommes s'imaginent dans l'exercice libéral, mais en outre, la plupart d'entre eux souhaitent avoir une approche globale de la naissance, c'est-à-dire intervenir de la déclaration de la grossesse jusqu'aux suites de couches et aux soins pédiatriques.

Cette description presque idéale de la profession est certes le fait d'élèves et non pas de professionnels établis. De même, on peut penser qu'il s'agit là d'un discours de personnes tentant de se rapprocher de la pratique des professionnels de la médecine qu'ils auraient aimé être, autrement dit un facteur révélant la difficulté pour

116 A Travail, Genre et Sociétés n 12 - Novembre 2004 
certains d'entre eux à faire leur deuil. Mais la profession de sage-femme n'est-elle pas officiellement une profession médicale et les sages-femmes ne souhaitent-elles pas être associées à ce statut dans les faits ? Autrement dit, en se positionnant de la sorte, ils ne se conforment pas à ce que l'activité professionnelle est, mais à ce qu'elle pourrait être dans l'opinion d'une bonne partie des sages-femmes françaises. Ce désir soutient donc le discours de ces futurs sages-femmes. Ils s'interrogent beaucoup sur l'évolution de la profession. Ainsi, il apparaît pour leur grande majorité que l'exercice libéral s'impose comme une réponse possible.

Ainsi, le fait d'être un homme prédisposerait à adopter une pratique professionnelle actuellement en marge statistiquement. Autrement dit, émerge une tendance à une différenciation dans les désirs professionnels de ces étudiants. Ils se distinguent donc doublement : d'une part leur genre les différencie, d'autre part leur conception de l'activité professionnelle les éloigne des réalités professionnelles d'une grande majorité des sages-femmes ${ }^{22}$.

Cela peut expliquer leur propension à être critiques envers les sages-femmes en poste, même celles qui exercent en libéral. L'inadéquation avec la pratique du suivi global, sorte de type idéal de la profession, au sens wébérien du terme, et pour lequel ils fondent beaucoup d'espoir, est pour eux un critère discriminant.

\section{L'atout du genre masculin pour la valorisation de la profession}

L'association forte entre ces hommes et un avenir professionnel passant par l'exercice libéral fait penser que leur genre peut être finalement un atout dans leur insertion professionnelle, bien plus qu'un handicap. Ici, ce sont les représentations de la place des hommes dans une profession qui jouent en leur faveur.

En tant que représentantes d'une profession médicale, les sages-femmes veulent se positionner sur un pied d'égalité avec les médecins. Dans ce contexte, l'introduction d'hommes parmi les sages-femmes peut contribuer à émanciper la profession par rapport aux autres professions de santé et de l'univers médical. La logique de dévalorisation des professions qui se féminisent se trouve inversée, ce qui revient à vérifier à nouveau le mécanisme. De plus, en égratignant les compétences "féminines", certaines sages-femmes acceptent de reconnaître une compétence plus strictement professionnelle. Le rôle que les hommes entendent prendre dans la profession est autant un rôle qui répond à un habitus mascu-

\author{
22 A titre de \\ comparaison, on \\ rencontre la même \\ logique chez les \\ infirmiers : pour une \\ moyenne de $15 \%$ \\ d'activité libérale pour \\ toute la profession, on \\ recense $20 \%$ \\ d'hommes, c'est-à-dire \\ une sur-représentation \\ de ceux-ci. B. Bon- \\ Saliba, Les infirmières, \\ Paris, Syros, 1993.
}


lin qui veut que les hommes prennent en charge les plus grandes responsabilités au niveau d'une organisation (Bourdieu, 1998), qu'un rôle qui est subtilement suggéré par les personnes détentrices de pouvoir dans la formation, donc des femmes. Ainsi, un sage-femme établi à Paris, très engagé syndicalement, dit refuser régulièrement de nouvelles responsabilités que lui offrent ses collègues femmes. Le parallèle pourrait être fait avec les infirmiers qui sont en plus forte proportion que leurs collègues féminines dans des postes à responsabilités (enseignants, surveillants, cadres) ou bien techniques (Saliba, op.cit.).

Dès les premières années, mais surtout en troisième et quatrième année, les élèves sages-femmes se positionnent tous en faveur d'une véritable professionnalisation des sages-femmes. Au passage, cela débouche aussi sur des reproches envers leurs collègues femmes dont ils stigmatisent l'attentisme et le manque d'implication dans la vie du groupe professionnel mais aussi de leur service. Ces critiques permettent à ces garçons de se valoriser en faisant une distinction en fonction du genre et en instaurant une segmentation dans les représentations de ce que devrait être le groupe professionnel. Ils reproduisent le schéma selon lequel le fait d'être un homme prend mieux en compte les questions autour de la professionnalisation qu'une femme. Le masculin est pensé comme un atout, à la fois face à ce qui est perçu comme un défaut d'engagement des femmes sages-femmes, et face à l'argument de la conciliation des rôles familiaux et professionnels en ce qui concerne les femmes.

\section{Le rôle des responsables et la place des hommes dans la stratégie de professionnalisation}

Il a été abondamment démontré que l'arrivée de femmes dans une profession entraînait fréquemment un discours sur une dévalorisation du secteur. Marlaine Cacouault-Bitaud a montré qu'il s'agit bien souvent d'un moyen de passer sous silence les réels enjeux ou mutations au sein d'une profession (Cacouault-Bitaud, 2001). Or, certaines responsables de la profession déclarent librement que l'apport d'hommes assurera une meilleure reconnaissance du groupe professionnel. Elles ont en quelque sorte intégré, voire instrumentalisé, la logique de valorisation professionnelle par les hommes.

Les futurs sages-femmes sont d'autant plus enclins à accepter le rôle "d'agent de revalorisation" que celui-ci est relayé au sein de l'école. A ce titre, les enseignants jouent un rôle déterminant en aidant les étudiants, ne serait-ce 
qu'à trouver leur place dans le système professionnel. Ces derniers sont conscients du rôle qu'on voudrait leur attribuer. Cette dynamique, ils tentent aussi de la nommer. Nous allons voir qu'à titre d'explication, ils font tour à tour référence aux personnalités, aux bienfaits de la mixité professionnelle ou bien à une stratégie raisonnée des autorités professionnelles qui voient dans l'apport d'hommes un élément valorisant la profession.

La directrice de l'Ecole est régulièrement présente dans le discours des étudiants: "Madame la directrice, c'est une militante active pour les hommes" explique Stéphane. Selon lui, il en va de même pour l'équipe enseignante: "Sylvie [la monitrice de la promotion de l'élève] elle nous a toujours appréciés". Ces dernières prêtent une attention particulière aux "recrues" masculines, surtout dans l'entretien d'accueil, sans doute pour dissiper tout de suite les doutes chez les candidats. Pour expliquer cette attitude, et l'ouverture voulue de la formation aux garçons, certains insistent sur la question de la mixité sociale et professionnelle. Ils tentent ainsi de relier leur position très marginale avec une dynamique professionnelle plus large.

"Dans l'Ecole, il y a que des femmes, que ce soit la secrétaire, la directrice, les monitrices, la femme de ménage. Donc elles sont entre elles. Bon elles ont des rapports de femmes à femmes, $c^{\prime}$ est des groupes de femmes et le fait $q u^{\prime} i l$ y ait un homme là-dedans, ben c'est... Parfois ça les dérange, parfois ça... Je crois qu'elles sont contentes; ça leur donne une certaine idée de la mixité...", Pierre, quatrième année.

Pour ces étudiants, leurs responsables ont pour stratégie d'ouvrir la profession aux hommes dans l'objectif de la faire évoluer. Cela aurait pour conséquence une remise en cause des compétences "dites féminines". Indirectement, ces garçons entérinent la dialectique de valorisation/dévalorisation, alors que l'enjeu de professionnalisation et de segmentation possible semble être la dynamique majeure à l'œuvre.

$\mathrm{Au}$ niveau de l'intégration professionnelle, il est évident que cette attitude joue en faveur des garçons. Si bien que certains ont même l'impression de bénéficier d'un véritable traitement de faveur.

"Les sages-femmes enseignantes, elles étaient assez favorables parce que de toute façon c'était une volonté de l'école aussi, enfin c'était une volonté de l'école de faire le recrutement par médecine. Et quand sont arrivés les garçons, et ben j'ai pas eu l'impression que ça leur posait de problème. J'ai plutôt l'impression qu'elles s'y sont bien adaptées, par rapport à d'autres écoles... (...) Enfin, elles nous ont acceptés assez facilement. Il y avait même un laxisme relatif à notre égard par rapport aux filles. Je veux dire $q u^{\prime}$ on arrivait en retard ou un cours sautait par-ci, par-là... De principe, (...) on se faisait engueuler. Mais j'avais l'impression que c'était moins 
agressif, moins sérieux que quand c'était une fille qui faisait ça. Là, c'est mon sentiment là-dessus", Sylvain, quatrième année.

Cette stratégie, nous l'avons retrouvée d'une manière détournée dans le discours d'une sage-femme qui participe au recrutement dans la maternité grenobloise. A compétence et expérience équivalentes, elle déclare que son choix se portera plutôt sur un homme que sur une femme. Elle justifie son opinion par des raisons de gestion du service. En effet, la proportion de femmes sagesfemmes exerçant à temps partiel est majoritaire dans son service. A ce titre, elle pense qu'un homme s'impliquera plus dans son travail car il acceptera de travailler à temps plein. Cet exemple montre combien l'incorporation d'une "dose" d'hommes dans la profession peut apparaître, pour un cadre de la profession, comme une solution très concrète aux difficultés de gestion d'un service de maternité. Il démontre aussi que l'intégration des hommes est un enjeu pour l'organisation du métier.

\section{La proximité des étudiants avec les enjeux professionnels}

Il convient de souligner la connaissance que possèdent ces étudiants des enjeux et débats qui traversent la profession. A cet égard, l'implication des étudiants est d'autant plus importante qu'ils sont proches du diplôme. D'une manière générale, ils sont extrêmement attentifs à leurs conditions de travail et à la façon d'exercer.

Certains discours expriment un certain désarroi face aux débats en cours dans la profession. Notamment, ces futurs sages-femmes ne veulent pas que des contraintes organisationnelles, politiques ou simplement liées à l'exercice d'un travail salarié puissent les détourner de leur idéal, c'est-à-dire l'accompagnement global de la grossesse.

Cette attitude, au-delà des conditions d'exercice, émerge principalement au sein de deux thèmes, deux éléments de débats dans la profession: le rapport des sages-femmes aux médecins, la question de l'usage de la péridurale.

${ }^{23}$ Soulignons que la profession de gynécologuesobstétriciens tend à se féminiser, comme la plupart des spécialités médicales. En 2000, il y avait $35 \%$ de femmes dans cette profession. (DREES, 2001).
Les rapports avec les médecins sont primordiaux car on touche à l'exercice du pouvoir dans les maternités. Conscients qu'ils devront se confronter à des médecins encore majoritairement des hommes ${ }^{23}$, ils seront potentiellement en concurrence, car la présence d'hommes chez les sages-femmes est une information peu répandue. Ce débat est donc un lieu privilégié pour participer à leur intégration, en défendant l'indépendance des sages- 
femmes. Pour cela, ils peuvent convoquer l'outil légal (le code de déontologie), mais aussi un temps des origines où la sage-femme avait une réelle indépendance, ou encore des revendications teintées de corporatisme.

\begin{abstract}
"Les médecins sont pas au-dessus des sages-femmes, d'un point de vue de la hiérarchie. Comment dire?... Article 7 du code de déontologie de la sage-femme: "la sage-femme ne peut aliéner son indépendance professionnelle sous quelque forme que ce soit". Donc une sage-femme qui prend en charge mettons une femme qui vient accoucher, alors qu'elle est de garde, si le déroulement de l'accouchement de cette femme rentre dans le cadre qui est imposé par la loi sage-femme, c'est-à-dire une grossesse normale, un accouchement normal, physiologique - on peut utiliser les termes qu'on veut - la sage-femme elle a besoin de demander à personne ce $q u^{\prime}$ elle a à faire, et elle n'a d'ordre à recevoir de personne. Point. Donc là, la notion de hiérarchie elle existe pas. Je suis le seul maître à bord, avec la femme", Sylvain, quatrième année.

"Ben moi j'espère que ça évoluera dans ce sens là, qu'on trouve des solutions, pour que notre pratique redevienne plus ce qu'elle était à l'origine. Et que la sage-femme aussi garde son indépendance. Parce qu'en passant, la sage-fermme était complètement indépendante quand elle accouchait une femme chez elle, et quand elle est passée dans le milieu hospitalier, elles se sont retrouvées sous la tutelle du médecin, et donc à perdre leur indépendance, et à se retrouver finalement à faire presque entre guillemets du travail d'infirmière", Gabriel, troisième année.
\end{abstract}

Ce dernier extrait d'entretien met en avant des arguments corporatistes. Cependant, ils expriment bien la logique d'intégration sur laquelle s'appuient les étudiants. Ces raisonnements permettent d'incorporer véritablement le système professionnel, même s'il existe un risque de distinction par rapport aux femmes sagesfemmes. Pour ces étudiants, la logique corporatiste, convoquée dans les relations aux médecins, participe à leur intégration d'autant plus que cela leur permet d'alimenter la représentation de "l'accoucheur de la profession".

Les étudiants de première année sont plus nuancés, privilégiant la logique de coopération entre les professions.

"Non ça on la respecte la position hiérarchique, quand même. Mais vis-àvis des relations humaines, $c^{\prime}$ est pas parce qu'ils [les médecins] en savent plus qu'ils doivent nous traiter comme de la merde. Parce que bon, c'est hiérarchique mais on fait des métiers complémentaires. En tout cas nous on fait notre boulot et puis si on besoin d'eux on les appelle. C'est complémentaire", William, première année.

Ces étudiants suivent une voie discordante par rapport aux pratiques quotidiennes des sages-femmes. Cependant, elle est paradoxalement concordante dans le sens où ils rejoignent les désirs de la grande majorité des sagesfemmes à propos de réalités professionnelles à venir. Il est manifeste qu'à l'heure actuelle, ces élèves ont trouvé un 
moyen de contourner les compétences "féminines" et cette voie passe par une volonté affichée de professionnalisation de leurs pratiques. On serait tenté de penser que ce rôle " $d$ 'accoucheur de la profession" émergeant dans leurs discours, ira en s'atténuant avec la montée croissante et prévisible de leur nombre. En effet, l'arrivée des hommes coïncide avec l'acquisition progressive d'une reconnaissance médicale (une des conséquences du conflit de 2001) et, par conséquent, d'un effacement de l'imaginaire alimentant les compétences "dites féminines".

Ces étudiants se positionnent comme des éléments incitant leurs collègues à plus de professionnalisme. Ainsi, ils se situent dans une forte implication envers le groupe professionnel et la mise en forme de la distance professionnelle nécessaire pour l'exercice du métier. De ce fait, ils ont tendance à apparaître aujourd'hui en marge $\mathrm{du}$ reste des praticiennes, parce qu'ils viennent perturber les représentations professionnelles établies.

Même si objectivement ils profitent de la valorisation par le masculin, ces étudiants montrent que la question de la mixité chez les sages-femmes ne se pose pas en tant que telle. Ils ne sont pas des militants de la masculinisation de cette profession. Ils n'ont pas davantage l'impression $\mathrm{d}^{\prime}$ endosser un rôle de pionnier. Leur problème est bien de prouver aux yeux de tous qu'ils sont des sages-femmes à part entière et que leur intégration professionnelle donne lieu à très peu d'obstacles. C'est par ce souci d'intégration réussie qu'ils participent pleinement à un mouvement de professionnalisation des sages-femmes.

On peut penser que leur attitude - sans doute suivie par de plus en plus de sages-femmes si l'on en croit les revendications présentes lors du dernier conflit - pose aussi des questions sur les pratiques et les orientations que la majorité des sages-femmes femmes souhaitent donner à leur métier. Autrement dit, les hommes peuvent être des éléments déclenchant une réflexion des praticiennes sur leur propre travail. Par leur présence, ils rendent pertinent le débat interne à propos de l'avenir professionnel des sages-femmes. A ce titre, il conviendrait de poursuivre cette recherche en se penchant sur les pratiques professionnelles, à savoir : quelle est l'influence de l'introduction d'hommes dans un service de maternité ou dans un cabinet libéral, et existe-t-il des "faisceaux de tâches" (Hughes, 1996) proprement masculins ? 


\section{BIBLIOGRAPHIE}

ALONZO Philippe, 1996, Femmes employées. La construction sociale sexuée du salariat, Paris, L'Harmattan.

AUDRIC Sophie, NIEL Xavier, SICARD Daniel, VILAIN Annick, 2001,"Les professions de santé. Eléments d'informations statistiques", Revue solidarité et Santé, DRESS.

BOURDIEU Pierre, 1990, "La domination masculine", Actes de la Recherches en Sciences Sociales, $\mathrm{n}^{\circ} 84$, pp.4-31.

BOURDIEU Pierre, 1998, La domination masculine, Paris, Le Seuil.

BOIGEOL Anne, 1993, "La magistrature française au féminin : en spécificité et banalisation", Droit et Société, vol. 25, pp.489-523.

BUCHER Rue et STRAUSS Anselm, "Profession in process", in A. STRAUSS, 1992, La trame de la négociation, Paris, L'Harmattan, pp.67-86

CACOUAUlt Marlaine, 1998, "Variations sur les métiers féminins et masculins", in M. MARUANI (dir.), Les nouvelles frontières de l'inégalité, Paris, La Découverte, p.20.

Cacouault-Bitaud Marlaine, 2001, "La féminisation d'une profession estelle un signe de baisse de prestige ? ", Travail, genre et sociétés, n 5 , pp.93115 .

CARRICABURU Danièle, 1987, La profession de sage-femme: de la compétence limitée à l'autonomie relative, Mémoire de DEA, Paris, EHESS.

CARRICABURU Danièle, 1994, "Les sages-femmes face à l'innovation technique", in P. AÏACH et D. FASSIN, Les métiers de la santé, Paris, Anthropos, pp.281-308

COLLIN Johanne, 1994, "Féminisation, mutation professionnelle et transformation d'une identité collective. Le cas de la pharmacie au Québec", in C. DUBAR, Y. LUCAS, Genèses et dynamiques des groupes professionnels, Lille, PUL, pp.171-180.

DUBAR Claude, 1996 (1991), La socialisation. Construction des identités sociales et professionnelles, Paris, Colin.

DUBAR Claude, Tripier Pierre, 1998, Sociologie des professions, Paris, Armand Colin.

GADREY Nicole, 1992, Hommes et femmes au travail. Inégalités, différences, Identités, Paris, l'Harmattan.

GELIS Jacques, 1988, La sage-femme ou le médecin, Paris, Fayard.

HuGHES Everett C., 1996, Le regard sociologique, textes réunis par J. M. Chapoulie, Paris, éd. EHESS.

HUGHES Everett C., 1996, "Les professions établies", in Le regard sociologique (textes réunis par Jean Michel Chapoulie), Paris, EHESS, pp. 107-121.

LAGET Mireille, 1982, Naissances - L'accouchement avant l'âge de la clinique, Paris, Le Seuil.

MARRY Catherine, 1989, "Femmes ingénieurs : une (ir)résistible ascension ?", Information sur les sciences sociales, vol. 28, $\mathrm{n}^{\circ} 2$, pp.291-344

MARRY Catherine, 1992, "Femme et ingénieur : la fin d'une incompatibilité ?", La recherche, $\mathrm{n}^{\circ} 241$.

MARRY Catherine, 1994, "Les femmes ingénieurs: au-delà de l'antinomie entre le métier et la carrière, la famille", Cahiers du GEDISST, $\mathrm{n}^{\circ} 11$.

MuEL-DREYFus Francine, 1983, Le métier d'éducateur, Paris, Seuil. 
PARADEISE Catherine, 1985, "Rhétorique professionnelle et expertise", Sociologie du travail, $\mathrm{n}^{\circ} 1$, pp.17-31.

POUVREAU-ROMILly Odette Yvonne, 1991, L'événement de la naissance, Toulouse, Eres.

SAliBA Jacques, BON-SALIBA Brigitte, OUVRY-VIAL Brigitte, 1993, Les infirmières, Paris, Syros.

SCHWEYER François-Xavier, "La profession de sage-femme : autonomie au travail et corporatisme protectionniste", 1996, Sciences sociales et santé, vol. $14, \mathrm{n}^{\circ} 3, \mathrm{p} .68-100$.

124 \ Travail, Genre et Sociétés n 12 - Novembre 2004 